\title{
Actual practice of Kochi oxydol radiation therapy for unresectable carcinomas by intra-tumoral administration of hydrogen peroxide as a radiosensitizer
}

\author{
SHIRO OBATA, YUTAKA ISHIMARU, SHIGENORI MIYAGI, MIKA NAKATAKE, \\ AKIRA KUROIWA, YOSHIAKI OHTA, TSUNEHIKO KAN, SHINYA KANEGAE, \\ YOHTA INOUE, RHOICHI NISHIZATO and KOHKI MIYAZAKI
}

Department of Radiology and Radiotherapy, Nagasaki Prefecture Shimabara Hospital, Shimabara, Nagasaki 855-0861, Japan

Received September 24, 2021; Accepted November 24, 2021

DOI: $10.3892 / \mathrm{mco} .2022 .2501$

\begin{abstract}
Kochi oxydol radiation therapy for unresectable carcinomas (KORTUC) is a novel cancer treatment method developed in Japan. KORTUC targets resistance factors in cancer therapy, such as low-oxygen environments and excessive antioxidant enzymes. This may enhance the effects of conventional treatments. The present study reports the experience of the Nagasaki Prefecture Shimabara Hospital in using KORTUC treatment for a series of 210 patients between January 2010 and June 2019. When this radiosensitizer, a mixture of a dilute hydrogen peroxide solution ( $0.5 \mathrm{ml}, 3 \% / \mathrm{unit})$ and sodium hyaluronate $(2.5 \mathrm{ml}, 0.83 \%$ /unit $)$, is administered and applied directly to the cancer lesion, antioxidant enzymes are neutralized and degraded causing reoxygenation as a secondary by-product, thereby enhancing the cytotoxic effect of radiation. The radiosensitizer was administered twice per week before irradiation. Up to June 2019, KORTUC was administered to 210 patients. The most common disease stage was stage IV in 137 patients (65\%), followed by stage III in 25 patients, stage I in 17 patients and stage II in 7 patients (unknown disease stage in 24 patients). Of the 186 patients who could be followed up after the treatment, 28 (15\%) patients had a complete response (Response Evaluation Criteria in Solid
\end{abstract}

Correspondence to: Dr Shiro Obata, Department of Radiology and Radiotherapy, Nagasaki Prefecture Shimabara Hospital, 7895 Shimokawajiri, Shimabara, Nagasaki 855-0861, Japan

E-mail: s.obata@nagasaki-hosp-agency.or.jp

Abbreviations: KORTUC, Kochi oxydol radiation therapy for unresectable carcinomas; RECIST, Response Evaluation Criteria in Solid Tumors; CTCAE, Common Terminology Criteria for Adverse Events; IMRT, intensity modulated radiation therapy; CT, computed tomography; OS, overall survival

Key words: Kochi oxydol radiation therapy for unresectable carcinomas, radiosensitizer, hydrogen peroxide, hypoxia, reoxygenation
Tumors version 1.1), 59 (32\%) had a partial response, $73(39 \%)$ had stable disease and $26(14 \%)$ had progressive disease. No significant treatment-related adverse events were observed. The present study highlights the reports of 4 cases ( 3 cases from among the 28 patients with complete responses): i) A case of advanced, inoperable breast cancer; ii) a refractory patient with recurrence a decade after postoperative irradiation; iii) a patient with advanced, inoperable rectal cancer; and iv) a patient with lymph node metastases. Overall, KORTUC showed good efficacy and tolerable safety for various types of radioresistant tumors, and it has the potential for immediate worldwide use.

\section{Introduction}

Kochi oxydol radiation therapy for unresectable carcinomas (KORTUC) is a novel cancer treatment method developed in Japan (1). Although low-oxygen environments and excessive antioxidant enzymes are known to promote resistance to cancer therapy, elimination of these factors is difficult and has been rarely reported. If these treatment resistance factors can be removed, it is expected that the effects of conventional therapies, such as radiation therapy and chemotherapy, will be enhanced. Tumor cells are resistant to X-ray irradiation due to their high peroxidase and catalase activities. Therefore, by providing exogenous hydrogen peroxide before irradiation, the activities of anti-oxidative enzymes can be blocked and oxygen molecules can be produced simultaneously, leading to oxidative damage to low-linear energy transfer (LET) radioresistant tumor cells. Through this mechanism, low-LET radioresistant tumor cells can be converted into highly radiosensitive cells (1-5) (Figs. 1 and 2). KORTUC was designed to accomplish this goal.

When a mixture of a dilute hydrogen peroxide solution and sodium hyaluronate is administered directly to a cancer lesion, antioxidant enzymes are neutralized and degraded, resulting in increased intratumor oxygen tension, thereby enhancing the cytotoxic effect of radiation $(1,6)$.

To date, a Japanese clinical study of KORTUC in patients with breast cancer who refused surgery (6) and a phase I study 
of KORTUC in patients with locally advanced breast cancer in the United Kingdom (7) have been conducted, both of which demonstrated safety and showed indications of marked treatment effects.

The present study summarizes the outcomes of KORTUC therapy for a case series of 210 patients and provides the case reports of 4 patients who failed standard treatment and were successfully treated with KORTUC, including a case of advanced, inoperable breast cancer, a refractory patient with recurrence a decade after postoperative irradiation, a patient with advanced, inoperable rectal cancer and a patient with lymph node metastases.

\section{Materials and methods}

Target patients. In the hope that KORTUC would enhance the effects of existing treatments, from January 2010, following approval of the Nagasaki Prefecture Shimabara Hospital Ethics Committee (Shimabara, Japan; approval no. 21SH103 from January 14, 2010), Nagasaki Prefecture Shimabara Hospital included the following patients with difficult-to-control tumors as patients eligible for KORTUC: i) Patients resistant to radiotherapy; ii) patients with repeated recurrences; and iii) patients resistant to standard therapy. In September 2014, after confirmation of its efficacy and safety in 62 patients, the following further patient groups were added with approval from the Ethics Committee (additional approval no. 26SH185 from September 4, 2014): iv) Patients who refused surgery and v) patients requiring multidisciplinary therapy. The present study targets solid tumors of all cancer types in which a sensitizer can be administered intratumorally. Considering the desire of each patient for KORTUC and the need for the treatment, all patients provided written informed consent before being included in the study. The study was conducted following the Declaration of Helsinki of the World Medical Association.

Treatment method. The radiosensitizer consisted of 1 unit $(0.5 \mathrm{ml})$ of a $3 \%$ hydrogen peroxide solution mixed with $2.5 \mathrm{ml}$ of $0.83 \%$ sodium hyaluronate, resulting in a $0.5 \%$ hydrogen peroxide concentration. The actual injected dose was determined so that a reaction would occur as evenly as possible over the entire lesion. For example, for a lesion of $1 \mathrm{~cm}$ in diameter, the injected dose of the mixture was $\sim 1 \mathrm{ml}$, and for a lesion $3 \mathrm{~cm}$ in diameter, the injected dose of the mixture was $\sim 3 \mathrm{ml}$.

The addition of sodium hyaluronate stabilized the hydrogen peroxide and resulted in elevated intratumor oxygen tension even $48 \mathrm{~h}$ after injection (8). Thus, the radiosensitizer was administered twice per week. To maximize its sensitizing effect, the radiosensitizer was injected intratumorally immediately prior to radiation whenever possible; for example, on Monday and Wednesday or on Tuesday and Thursday, depending upon the radiation schedule (this injected form of KORTUC is known as KORTUC II). For a lesion located on the skin surface, the radiosensitizer was sprayed over the lesion, or a gauze pad soaked in the radiosensitizer was applied to the lesion just before radiation (this form of KORTUC is known as KORTUC I). For a deep-seated lesion, the radiosensitizer was injected slowly into the tumor with an 18- to 26-G needle under ultrasound or computed tomography (CT) guidance to avoid an accidental puncture of adjacent vessels or organs. As KORTUC II is a novel treatment, there have been no data on the risk of tumor cell dissemination. For this reason, a needle with the smallest possible diameter, depending on the conditions, was selected based on reports suggesting that the dissemination risk was $0.04 \%$ [10/25,000 cases (unclarified cancer type) in a systematic review of data up until the end of 1976] for a lung biopsy (9) under CT guidance and that a smaller puncture needle was associated with less frequent dissemination (10). The needle used most frequently was a 22-G spinal needle that was readily visible under imaging guidance and relatively flexible and hard to break. If a patient complained of severe pain, $1 \mathrm{ml}$ of $1 \%$ lidocaine was mixed with 1 unit of the radiosensitizer after confirming that the patient had no Xylocaine allergy.

Treatment responses were assessed according to the Response Evaluation Criteria In Solid Tumors (RECIST), version 1.1 (11). RECIST is a standard way to measure how well a patient with cancer responds to treatment; it is based on whether tumors shrink, stay the same size or increase in size. To use RECIST, there must be at least one tumor that can be measured on X-ray, CT or magnetic resonance imaging scans. The types of response a patient can have are a complete response (CR), a partial response (PR), progressive disease (PD) and stable disease (SD).

The survival period was calculated using the Kaplan-Meier method from the planned radiation therapy date to the date of death due to all causes or the date of the final follow-up of surviving patients (up to June 2019).

Adverse events were assessed according to the Common Terminology Criteria for Adverse Events (CTCAE), version 4 (12). CTCAE is widely accepted as the standard classification and severity grading scale for adverse events in cancer therapy, clinical trials, and other oncology settings.

\section{Results}

Treatment results. Between January 2010 and June 2019, KORTUC was administered to 210 patients. Table I shows the responses of the patients according to cancer type and stage. The most common disease stage was stage IV in 137 patients $(65 \%)$, followed by stage III in 25 patients, stage I in 17 patients and stage II in 7 patients (unknown disease stage in 24 patients). As most patients were in stage IV and radiotherapy was a local treatment, their post-treatment evaluation was assessed based on local effects and not on overall survival (OS) or disease-free survival. Of the 186 patients who could be followed up after the treatment, according to RECIST version 1.1, 28 (15\%) patients had a CR, 59 (32\%) had a PR, $73(39 \%)$ had SD and $26(14 \%)$ had PD. In addition, the median survival time of all $28 \mathrm{CR}$ patients was 30 months (range, 2-96 months), and the 2-, 3-, and 5-year OS rates were $83.1,76.2$ and $66.7 \%$, respectively. Table II shows the adverse events associated with KORTUC, which were assessed according to CTCAE version 4, and included the following: Radiation dermatitis, grade III in 6 patients and grade II in 76 patients; leukopenia, grade III in 2 patients (receiving concurrent chemotherapy); postoperative recurrence of perforation at the intestinal anastomotic site, grade IV in 1 patient; oral mucositis, grade III in 2 patients; radiation esophagitis, grade II in 22 patients; 


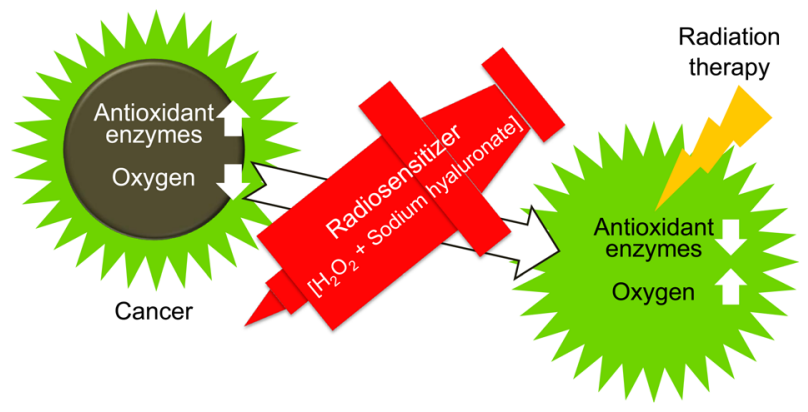

Figure 1. Method of Kochi oxydol radiation therapy for unresectable carcinomas, modified from Figure 4 published in reference (1).

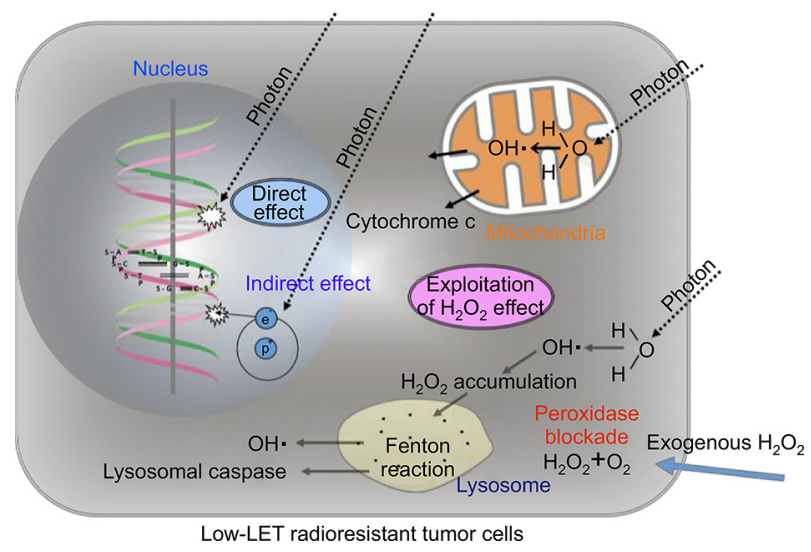

Figure 2. Molecular mechanism of Kochi oxydol radiation therapy for unresectable carcinomas. Permission was obtained for reuse of this image (5). LET, low-linear energy transfer.

radiation enteritis, grade II in 3 patients; and pain during injection of the radiosensitizer, grade I in 20 patients.

Representative case reports. The following are case reports of 4 patients who had failed conventional therapy but maintained a CR following KORTUC.

Case 1: Advanced and inoperable breast cancer. A woman in her 50s noticed a lump in the right breast, which was left untreated. The patient presented to a local doctor 1 year later with gradually worsening shortness of breath and lower extremity edema accompanied by anemia, and was diagnosed with stage IV breast cancer [invasive ductal carcinoma, papillotubular to scirrhous carcinoma; lymph node, liver and lung metastases; immunohistochemical examination (IH) result: $\operatorname{ER}(3+), \operatorname{PgR}(+)$ and HER2(3+)]. The patient responded inadequately to seven courses of $840 \mathrm{mg}$ pertuzumab/420 mg trastuzumab/100 mg docetaxel, seven courses of $200 \mathrm{mg}$ trastuzumab emtansine, $330 \mathrm{mg}$ trastuzumab/1,800 mg gemcitabine and fulvestrant for $\sim 3$ years. The primary lesion gradually grew into a giant lesion, $15 \mathrm{~cm}$ along its major axis, and was elevated enough to break through the skin. The tumor was hemorrhagic, had white exudate on its surface and had an unusually offensive odor. The breast cancer was resistant to treatment, and an experienced radiation oncologist in the same hospital was consulted about administering radiation therapy to the patient. It was considered that radiotherapeutic efficacy against the tumor progression could not be expected either. The patient was then referred to Nagasaki Prefecture Shimabara Hospital for KORTUC in August 2019 at the age of 56. Standard therapy had previously been used, but no treatment resistance factors had been identified. Therefore, it was considered that previous treatments had not been used effectively.

The patient now received intensity modulated radiation therapy (IMRT) with 44 Gy in 16 fractions for 3 weeks to the breast and the regional lymph nodes, followed by boosts of $9 \mathrm{~Gy}$ in three fractions to the primary lesion and involved ipsilateral lymph nodes. In addition, twice weekly, the patient received radiosensitizer injected into the breast cancer and multiple regional metastatic lymph nodes within the irradiation field. Furthermore, a hydrogen peroxide solution was sprayed on the surface of the tumor (KORTUC I \& II) (Fig. 3).

No serious adverse events were observed. At 4 months after KORTUC, the large mass and swollen lymph nodes within the radiation field regressed. A concavity was present on the right chest wall where the lesion had been present, but the size of the concavity reduced over time (Fig. 4). After 14 months, the patient succumbed to cancer, but no regrowth of the right chest wall lesion was observed (however, it should be noted that this case is not included in the aforementioned data due to the collection period).

Case 2: A refractory patient with recurrence a decade after postoperative irradiation. A woman in her 40 s noticed a lump in the left breast and underwent breast conserving surgery and axillary lymph node dissection for left breast cancer [invasive ductal carcinoma; n(+); IH: ER(+), PgR(+), HER2(2+); FISH unknown], and radiotherapy with 50 Gy in 25 fractions, at the Nagasaki Prefecture Shimabara Hospital. This was followed by adjuvant chemotherapy and endocrine therapy with tegafur/uracil and Nolvadex for the remaining breast tissue post-surgery. The patient voluntarily stopped visiting the Outpatient Department after 5 years. At 10 years after the first visit, irregular erosions and an ulcer, $10 \mathrm{~cm}$ long along its major axis and surrounded by multiple red nodules, developed in the remaining breast (Fig. 5). Metastasis was confirmed by skin biopsy [IH: ER(+), PgR(-), HER2(3+); Ki-67 34\%]. Femara was administered and then substituted with $1 \mathrm{mg}$ anastrozole per day, and 16 courses of trastuzumab therapy were administered, once every 3 weeks (first course of $342 \mathrm{mg}$ and then $252 \mathrm{mg}$ ). Despite these treatments, the disease worsened, and KORTUC with irradiation of 45 Gy in 25 fractions was performed only at the site of recurrence in February 2015 at the age of 53 .

Acute-phase grade III dermatitis was observed. The local lesion disappeared, with no serious adverse events after 6 years. During that time, bone metastasis and lung metastasis were observed, and adjuvant chemotherapy in the form of nine courses of $840 \mathrm{mg}$ pertuzumab/252 $\mathrm{mg}$ trastuzumab and $152 \mathrm{mg}$ trastuzumab emtansine (once every 3 weeks), and $4 \mathrm{mg}$ zoledronic acid hydrate (once every 4 weeks) was administered. A solitary small cerebellar metastasis was treated with stereotactic brain irradiation. The re-irradiated left chest wall lesion showed partial ulcer formation 4.5 years after KORTUC, and was accompanied by secondary infection. Cytology was class III, but has since improved. 


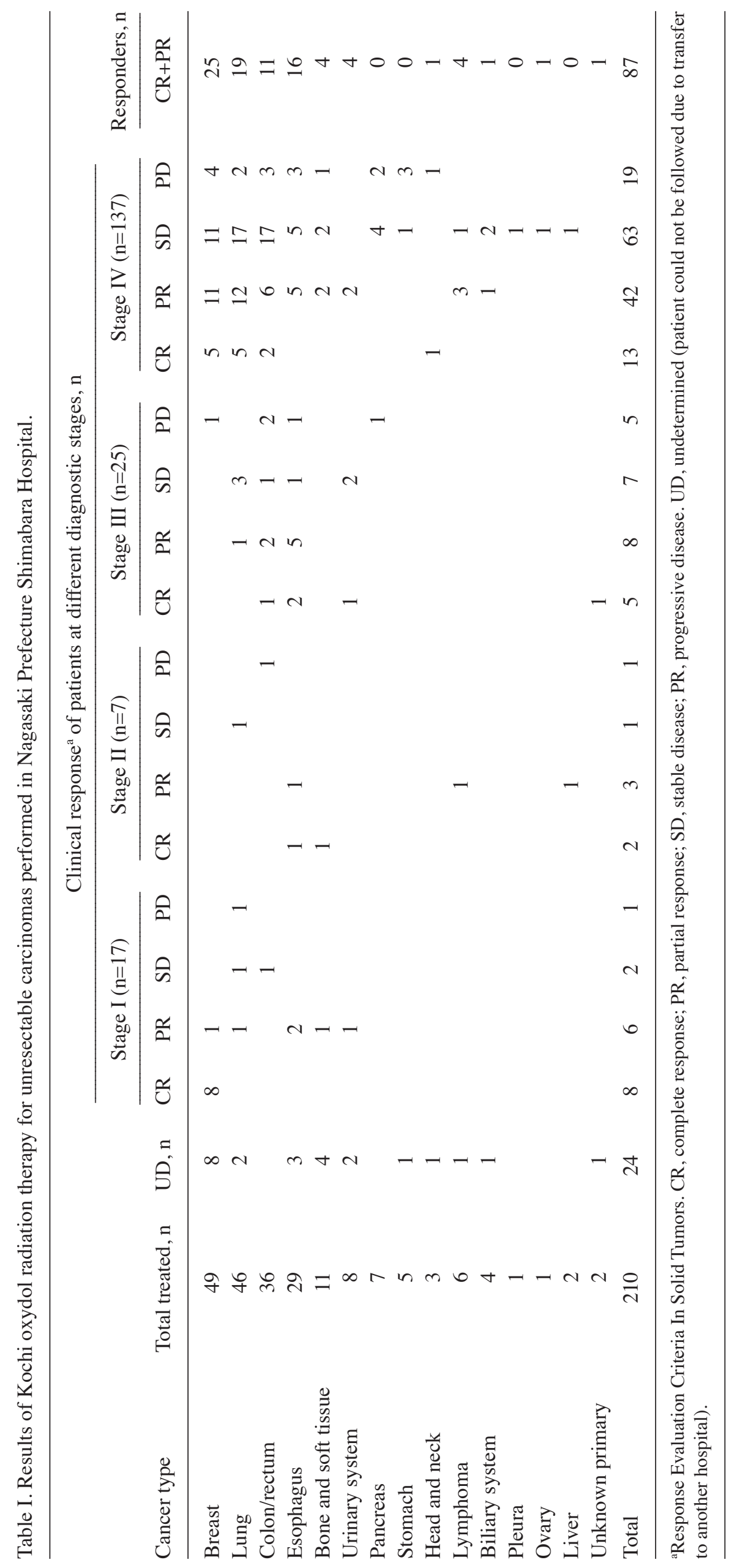


Table II. Adverse events following Kochi oxydol radiation therapy for unresectable carcinomas according to Common Terminology Criteria for Adverse Events version $4(n=210)$.

\begin{tabular}{lcr}
\hline Adverse Events & $\begin{array}{c}\text { Severity } \\
\text { (Grade) }\end{array}$ & $\begin{array}{r}\text { Number } \\
\text { of cases }\end{array}$ \\
\hline Radiation dermatitis & II & 76 \\
Radiation esophagitis & III & 6 \\
Pain during injection of the & II & 22 \\
radiosensitizer & I & 20 \\
$\begin{array}{l}\text { Radiation enteritis } \\
\text { Leukopenia (receiving concurrent }\end{array}$ & II & 3 \\
$\begin{array}{l}\text { chemotherapy) } \\
\text { Oral mucositis }\end{array}$ & III & 2 \\
$\begin{array}{l}\text { Postoperative recurrence of } \\
\text { perforation at the intestinal }\end{array}$ & IV & 2 \\
anastomotic site & & 1 \\
\hline
\end{tabular}

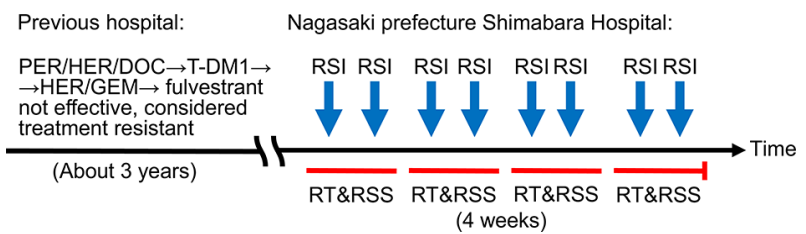

Figure 3. Case 1: Treatment flow of a patient with advanced and inoperable breast cancer. RT, radiation therapy (44 Gy in 16 fractions for 3 weeks and boosts of $9 \mathrm{~Gy}$ in 3 fractions for a week); RSI, radiosensitizer injection (into the primary and metastatic lymph nodes with ultrasonographic guidance); RSS, radiosensitizer spray (on the surface of the primary lesion); PER, pertuzumab; HER, trastuzumab; DOC, docetaxel; T-DM1, trastuzumab emtansine; GEM, gemcitabine.

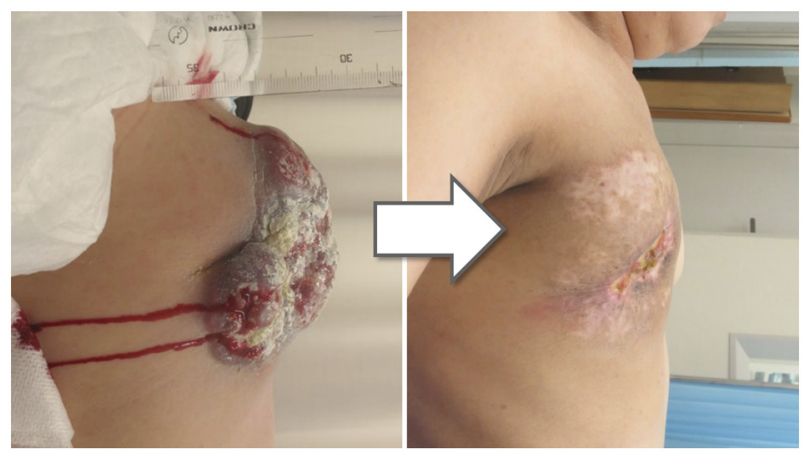

Figure 4. Images of the breast in case 1 prior to and at 4 months post-Kochi oxydol radiation therapy for unresectable carcinomas for treatment-resistant right-sided advanced breast cancer.

It has been reported that $\sim 10 \%$ of patients have local recurrence after standard treatment (13), suggesting that re-irradiated KORTUC may become an important salvage therapy for patients with recurrent disease.

Case 3: Advanced and inoperable rectal cancer. A 57-year-old female who visited the Emergency Outpatient Department of

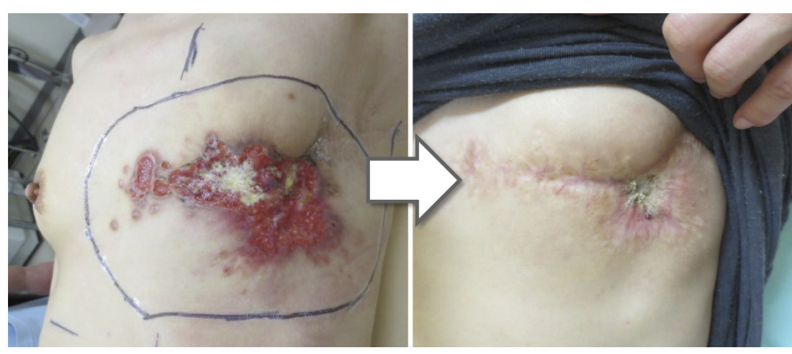

Figure 5. Case 2: Images of the breast showing scar tissue in place of left breast cancer recurrence at 38 months after salvage Kochi oxydol radiation therapy for unresectable carcinomas.

Nagasaki Prefecture Shimabara Hospital for left abdominal pain, appetite loss and nausea was diagnosed with rectal cancer at stage IVA, T4bN2aM1a, as a result of an inpatient examination in August 2015. The Eastern Cooperative Oncology Group performance status (PS) (14) was 3. The patient was confined to bed, with decreased appetite, severe anemia (hemoglobin: $6.5 \mathrm{~g} / \mathrm{dl}$; normal range, 11.6-14.8 g/dl), and weight loss of $10 \mathrm{~kg}$ over half a year. The patient had a type 2 tumor (moderately differentiated adenocarcinoma), which bleed regularly and was covered with white exudate, invading the total circumference of the lower rectum and extending to the perineum beyond the anal canal (Fig. 6). A CT scan revealed a partially unclear border with the uterus, suggestive of infiltration, and multiple perirectal, left internal iliac, left obturator, left external iliac and left inguinal lymph nodes of various sizes up to $2 \mathrm{~cm}$, strongly suggestive of lymph node involvement (Fig. 6). Based on these findings, the patient was informed that they had an inoperable cancer and a life expectancy of 2 weeks to 1 month, and it was recommended that best supportive care should be administered for symptomatic relief, instead of aggressive curative treatment. The patient was also reluctant to undergo curative therapy. However, the patient's family could not understand the sudden declaration of terminal cancer and consulted a gastroenterologist. This doctor also recommended palliative care, as chemotherapy could further shorten the lifespan of the patient. The family was still not satisfied with this recommendation and was eventually referred to the Department of Radiology and Radiotherapy, Nagasaki Prefecture Shimabara Hospital. Based on a discussion with the patient and their family, the patient was scheduled to receive KORTUC in combination with chemotherapy instead of standard treatment.

The patient underwent IMRT with 50.4 Gy in 30 fractions for 6 weeks to the primary lesion and regional lymph nodes, in the prone position using a belly board device. This was combined with twice-weekly injections and spray of the radiosensitizer on/into the perineal lesion, and use of both methods on/into the rectal lesion with endoscopic guidance. Two courses of modified FOLFOX6 at reduced doses (90\% doses; $241 \mathrm{mg}$ leucovorin and $103 \mathrm{mg}$ oxaliplatin over $2 \mathrm{~h}$ of continuous intravenous infusion, respectively; $482 \mathrm{mg}$ fluorouracil intravenously by slow bolus over $5 \mathrm{~min}$, and 2,894 mg fluorouracil over $46 \mathrm{~h}$ of continuous intravenous infusion) were administered concomitantly with radiation therapy. In addition, immediately before treatment initiation, a central venous port was placed, and intravenous hyperalimentation was initiated to improve the poor general condition 


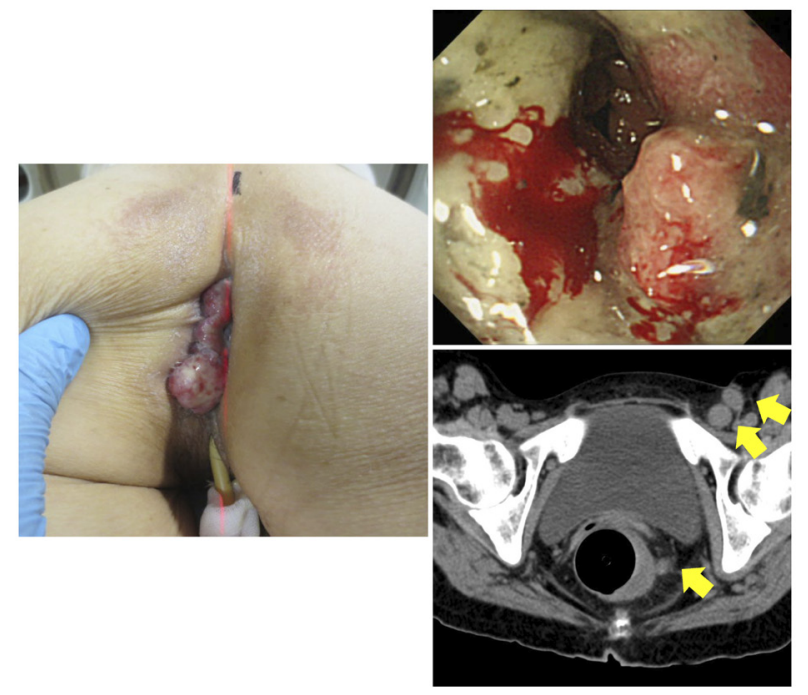

Figure 6. Case 3: Images and computed tomography scan of an inoperable case of advanced rectal cancer, stage IVA, modified from Figure 3 published in our previous study (15), with the consent of Jpn J Clin Radiol.

of the patient, to reduce adverse events and to increase the therapeutic effect. A colostomy was placed proximally to the descending colon to prepare for the development of ileus secondary to transient swelling caused by irradiation of the lower rectum.

At 9 months post-KORTUC, the rectal lesion had disappeared completely and an endoscopic rectal biopsy revealed no malignancy. The tumor extending to the perineum had disappeared. The perineal dermatitis was mild, possibly due to the low dose administered, and no serious adverse events were observed. The multiple lymph node metastases located on the left side of the pelvis had also regressed and could not be identified (Fig. 7). These included metastatic lymph nodes within the radiation field that had not been injected with the radiosensitizer.

This phenomenon has also been observed in other patients undergoing KORTUC. A possible explanation is that, due to its low molecular weight, the radiosensitizer injected into the primary lesion flows via the lymphatic drainage into the regional metastatic lymph nodes, similar to the way a dye or radioisotope is injected for sentinel lymph node identification in breast cancer, thereby sensitizing even lymph nodes that have not been injected. We named this phenomenon the 'sentinel effect' of KORTUC, as it is like the method of sentinel lymph node identification in breast cancer (15).

Elevated pre-treatment levels of carcinoembryonic antigen (264.5 ng/ml; normal range, 0-5 ng/ml) and carbohydrate antigen 19-9 (117 U/ml; normal range, 0-37 U/ml) returned to normal rapidly after KORTUC. Subsequently, the patient received adjuvant chemotherapy, 11 courses of mFOLFOX6 once every 3 weeks, and changing to 2 nd line therapy (FOLFIRI, 90\% dose; $300 \mathrm{mg}$ levofolinate calcium and $230 \mathrm{mg}$ irinotecan hydrochloride hydrate over $2 \mathrm{~h}$ of continuous intravenous infusion, respectively; $600 \mathrm{mg}$ fluorouracil intravenously by slow bolus over $5 \mathrm{~min}$, and $3,600 \mathrm{mg}$ fluorouracil over $46 \mathrm{~h}$ of continuous intravenous infusion), periodically for 4.5 years until the appearance of adverse events in the form of skin ulcers. The patient achieved

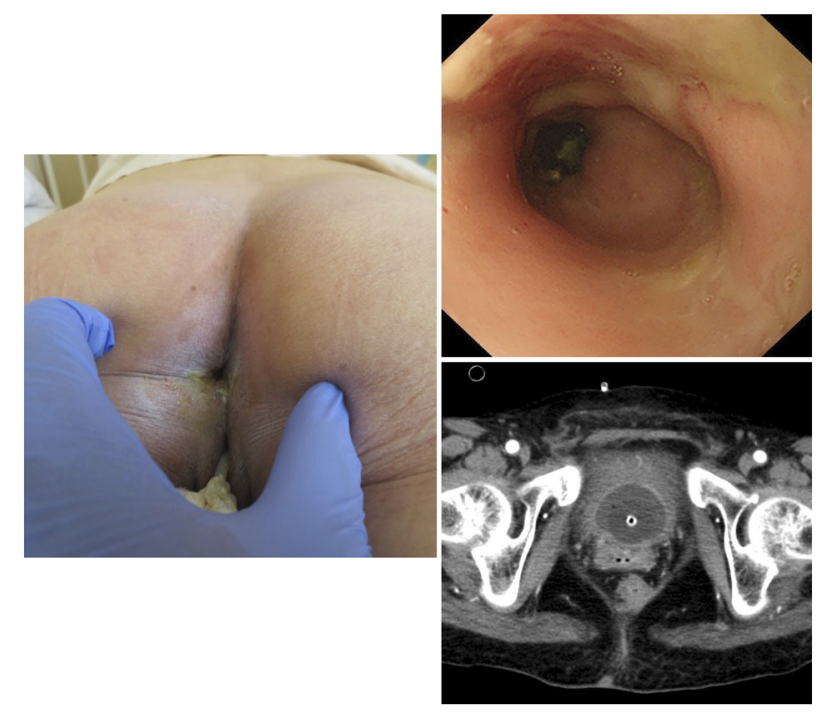

Figure 7. Images and computed tomography scan of case 3 at 9 months after Kochi oxydol radiation therapy for unresectable carcinomas and mFOLFOX6, modified from Figure 5 published in our previous study (15), with the consent of Jpn J Clin Radiol.

and has continued to achieve a CR, with normal weight and general overall condition for 6 years now without adjuvant cancer therapy. Currently, the patient is followed up once a month by the referral gastroenterologist.

Case 4: A huge lymph node metastasis. A 69-year-old male presented with advanced non-small cell lung cancer (NSCLC; RLL, adenocarcinoma, cT1bN3M1c, stage IVB; PS: 2) in September 2017. A left neck lesion appeared 1.5 months previously and increased in size rapidly to $12 \mathrm{~cm}$ along its major axis (Fig. 8). Due to the lesion, the patient complained of neck pain and numbness of the left upper extremity. The patient could not rotate their neck or elevate the ipsilateral upper arm, and developed Horner's syndrome. At first, the primary lesion was unknown; however, as a result of subsequent pathological findings, which suggested adenocarcinoma of the lung, and finding of a small nodule in the right lower lung on whole-body CT, this was considered to be the primary lesion. The tumor was consistent with lymph node metastasis and involved surrounding blood vessels, so was considered difficult to surgically resect. Radiation therapy could also have caused the rupture and collapse of blood vessels, and sudden changes. However, if left untreated, the tumor was expected to grow more rapidly and invade the surrounding tissues and blood vessels more widely, breaking through the skin, causing secondary infections and fatal bleeding. Impaired quality of life could then result through severe pain and motor dysfunction progression, followed by sudden death. Therefore, the patient was referred from the Department of Respiratory Medicine, Nagasaki Prefecture Shimabara Hospital, for palliative radiotherapy. It was decided that the patient should be treated using IMRT, with 40 Gy in 16 fractions for 3 weeks to the entire left neck lymph node lesion, followed by boosts of $9 \mathrm{~Gy}$ in three fractions to the shrinking lesion, in combination with twice-weekly injection of the radiosensitizer under ultrasound guidance, and $60 \mathrm{mg}$ oral S-1 twice a day for 3 weeks, withdrawn for 2 weeks. 


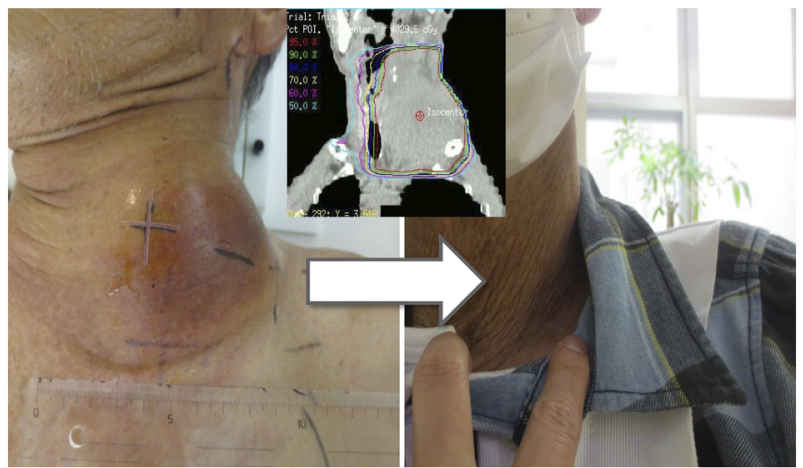

Figure 8. Case 4: Images and computed tomography scan (coronal image of dose distribution) of bulky lymph node metastases from non-small cell lung cancer prior to and at 8 months post-Kochi oxydol radiation therapy for unresectable carcinomas.

At 8 months after KORTUC initiation, the lesion disappeared, and concomitant conditions such as neck pain, motor dysfunction and Horner's syndrome subsided. Subsequently, the patient received adjuvant chemotherapy at the aforementioned dose periodically for 2 years, and no tumor regrowth was present before the patient succumbed to aspiration pneumonia.

Original abilities of radiation therapy. The adaptive size of lesions in stereotactic irradiation, which aims to have a therapeutic effect on cancer using large doses, is generally $\leq 5 \mathrm{~cm}$, and it is difficult to expect a curative effect on lesions of larger sizes. The combined use of the new sensitizer has shown a marked improvement in lesions with a size of $\geq 10 \mathrm{~cm}$ without increasing the total dose of radiation.

\section{Discussion}

Tailor-made treatments that have selective effects on certain types of cancer have become popular, such as KYMRIAH (tisagenlecleucel) (16). KORTUC, which broadly eliminates the source of cancer, appears to swim against such current trends, as it sensitizes radiotherapy, so can be considered effective for basically any type of cancer. However, KORTUC does not require many biopsied tissue samples to determine potential selective treatment effects and has favorable cost performance, as it does not require the reagents and tests that would be needed for treatments with limited indications. These advantages reduce the financial burden on patients and healthcare providers, state healthcare systems, and ultimately, taxpayers.

As the pursuit of tailor-made treatments, in other words, the diversity concerning malignant transformation, is thought to be the same as the pursuit of the infinite, potential adaptability of organisms, the development of tailor-made treatments should overcome the complexity of malignant transformation. It might be beneficial to reconsider the use of a single approach to treat the underlying cause of all cancer types.

KORTUC mainly exerts its effects through reoxygenation. After reoxygenation has been achieved, almost all molecular-targeted drugs would theoretically show the same local effects as those observed when the drug was administered to the tumor. Although it is known that an improved low-oxygen environment or reoxygenation enhances treatment effects, attempts to achieve reoxygenation have failed for a long period. In an attempt to apply this approach to clinical settings, however, attention was paid to the tumor growth, invasion and metastasis, as well as to the growth factors involved in the acquisition of metastatic potential, such as vascular endothelial growth factor (VEGF), epidermal growth factor (EGF) and other molecules, which underlie the influence of hypoxia (17). Based on the assumption that inhibiting the action of these factors may at least put a brake on cancer progression, molecular-targeted therapy has been developed. VEGF, EGF and other molecules, which are targets for molecular-targeted therapy, are produced in response to stimulation by hypoxia-inducible factor (HIF)-1 $\alpha$. However, HIF-1 $\alpha$ is rapidly ubiquitinated and deactivated if the underlying low-oxygen environment is improved. KORTUC can increase oxygen tension in hypoxic intratumor environments and thus KORTUC therapy could theoretically result in a halt to further production of target factors such as VEGF and EGF for molecular-targeted drugs. This means that KORTUC could eradicate the target molecules of molecular-targeted therapy. KORTUC not only provides local treatment, but also reduces the risk of tumor growth and metastasis. In the future, molecular-targeted therapy may only need to be used in patients who already have distant metastasis (M1) or are at a high risk for distant metastasis.

The threat that reoxygenation poses to cancer cells also involves the cancer cell cycle and cancer stem cells. Cancer stem cells are also called dormant cells, a number of which show treatment resistance in the $\mathrm{G}_{0}$ phase (quiescent stage). Once the cancer stem cells switch from the quiescent stage to the proliferation stage, radiotherapy and chemotherapy could exert the same effects as those observed in normal cancer cells (18-21). Since oxygen concentration appears to be involved in the transition from the quiescent stage to the proliferation stage, reoxygenation would enable this transition. This means that KORTUC can activate cancer stem cells and reduce the risk of recurrence after treatment.

In healthy conditions, reactive oxygen species (ROS) harm the body, and the presence of antioxidant enzymes that neutralize ROS is therefore essential. However, once a carcinogenic state occurs, the role is reversed and ROS are needed to eliminate the cancer, but the ROS become ineffective if neutralized by antioxidant enzymes. To survive and protect itself from ROS attack, cancer utilizes this neutralization reaction and uses transcription factors, thereby excessively producing antioxidant enzymes $(22,23)$. In the process of achieving reoxygenation, KORTUC neutralizes the excessive antioxidant enzymes that interfere with treatment; therefore, it may enhance the effects of radiotherapy and chemotherapy (24). However, unlike radiotherapy, which has high penetrability, chemotherapy requires an angioarchitecture that can deliver anticancer drugs to cancer. The resistance factors of both radiation therapy and anticancer drugs may overlap, and it cannot be denied that sensitizers affect both. Furthermore, it is undeniable that sensitizers were intentionally used in anticipation of the sensitizing effects of both radiation therapy and chemotherapy, especially in refractory patients. A number of good outcomes have been recorded that were not achieved with conventional chemoradiotherapy. A number of patients with terminal diagnoses have been cured 
using KORTUC, and this is a fact that cannot be overlooked, as it suggests that KORTUC may exceed the limits of current treatments. Based on the present results, it is strongly hoped that clinical trials will be conducted and data will be gained at other facilities worldwide, and that a therapeutic effect will be achieved in patients who are suffering from intractable cancer. Hypoxia and excessive antioxidant enzymes are among the main factors conferring resistance to both radiotherapy and chemotherapy. By neutralizing excessive antioxidant enzymes in cancer cells with hydrogen peroxide, which is a readily available ROS, antioxidant enzymes disappear, and oxygen occurs as the end product. By combining the end product with the remaining excessive ROS, irradiation of this state acts as a devastating attack against cancer. This is the core principle of KORTUC action (25-27).

A common question asked is whether the damage to the surrounding normal tissues is increased by KORTUC. When the oxygen partial pressure is $>30 \mathrm{mmHg}$, the influence of irradiation reaches a plateau (28). ROS produces water and oxygen as end products. Therefore, excessive ROS (unreacted dilute hydrogen peroxide) is the only factor that may increase adverse events, and no obvious increase in the incidence of adverse events was recorded in the present study following the addition of KORTUC to the general treatment.

In conclusion, the KORTUC radiosensitization method showed good efficacy and tolerable safety for various types of radioresistant tumors, and it has the potential for immediate worldwide use.

\section{Acknowledgements}

Not applicable.

\section{Funding}

No funding was received.

\section{Availability of data and materials}

The datasets used and/or analyzed during the current study are available from the corresponding author on reasonable request.

\section{Authors' contributions}

SO, AK, YO and TK conceived and designed the study. All authors performed the treatments. SO, YIs, YIn, SM, MN, RN, $\mathrm{KM}, \mathrm{SK}$ and $\mathrm{AK}$ analyzed and interpreted the data. SO, AK, YIs, YIn, SM, SK and MN wrote, reviewed, and revised the manuscript. SO and AK confirm the authenticity of all the raw data. All authors read and approved the final manuscript.

\section{Ethics approval and consent to participate}

The present study involving human data was approved by the Ethics Committee of Nagasaki Prefecture Shimabara Hospital ( approval no. $21 \mathrm{SH} 103$ from January 14, 2010; and additional no. 26SH185 from September 4, 2014). All patients provided written informed consent prior to their inclusion in the study. The study was conducted in accordance with the Declaration of Helsinki of the World Medical Association.

\section{Patient consent for publication}

All patients included in the present study provided consent for participation and publication.

\section{Competing interests}

The authors declare that they have no competing interests.

\section{References}

1. Ogawa Y, Yamashita T, Masunaga S, Kariya S, Tokuhiro S, Aoyama N, Tsuzuki A, Yaogawa S, Kubota K, Nishioka A, et al: A new enzyme-targeting and radio-sensitization treatment; basics and clinical practice of KORTUC Shinoharashinsha Pub. Inc., Tokyo, 2015 (In Japanese).

2. Ogawa Y, Takahashi T, Kobayashi T, Kariya S, Nishioka A, Hamasato S, Moriki T, Seguchi H, Yoshida S and Sonobe H: Immunocytochemical characteristics of human osteosarcoma cell line HS-Os-1: Possible implication in apoptotic resistance against irradiation. Int J Mol Med 14: 397-403, 2004.

3. Kariya S, Sawada K, Kobayashi T, Karashima T, Shuin T, Nishioka A and Ogawa Y: Combination treatment of hydrogen peroxide and $\mathrm{X}$-rays induces apoptosis in human prostate cancer PC-3 cells. Int J Radiat Oncol Biol Phys 75: 449-454, 2009.

4. Ogawa Y, Takahashi T, Kobayashi T, Toda M, Nishioka A, Kariya S, Seguchi H, Yamamoto $\mathrm{H}$ and Yoshida S: Comparison of radiation-induced reactive oxygen species formation in adult articular chondrocytes and that in human peripheral $\mathrm{T}$ cells: Possible implication in radiosensitivity. Int J Mol Med 11: 455-459, 2003.

5. Ogawa Y: Paradigm shift in radiation biology/radiation oncology-exploitation of the ' $\mathrm{H}_{2} \mathrm{O}_{2}$ Effect' for radiotherapy using low-LET (Linear Energy Transfer) Radiation such as X-rays and high-energy electrons. Cancers (Basel) 8: 28, 2016.

6. Ogawa Y, Kubota K, Aoyama N, Yamanishi T, Kariya S, Hamada N, Nogami M, Nishioka A, Onogawa M and Miyamura M: Non-surgical breast-conserving treatment (KORTUC-BCT) using a new radio-sensitization method (KORTUC II) for patients with stage I or II breast cancer. Cancers (Basel) 7: 2277-2289, 2015.

7. Nimalasena S, Gothard L, Anbalagan S, Allen S, Sinnett V, Mohammed K, Kothari G, Musallam A, Lucy C, Yu S, et al: Intratumoral hydrogen peroxide with radiation therapy in locally advanced breast cancer: Results from a phase 1 clinical trial. Int J Radiat Oncol Biol Phys 108: 1019-1029, 2020.

8. Tokuhiro S, Ogawa Y, Tsuzuki K, Akima R, Ue H, Kariya S and Nishioka A: Development of a new enzyme-targeting radiosensitizer (KORTUC) containing hydrogen peroxide for intratumoral injection for patients with low linear energy transfer (LET) radioresistant neoplasms. Oncol Lett 1: 1025-1028, 2010.

9. Christensen ES: Iatrogenic dissemination of tumor cells-dissemination of tumor cells along the needle track after percutaneous, transthoracic lung biopsy. Dan Med Bull 25: 82-87, 1978.

10. Sinner WN and Zajicek J: Implantation metastasis after percutaneous transthoracic needle aspiration biopsy. Acta Radiol Diagn (Stockh) 17: 473-480, 1976.

11. Eisenhauer EA, Therasse P, Bogaerts J, Schwartz LH, Sargent D, Ford R, Dancey J, Arbuck S, Gwyther S, Mooney M, et al: New response evaluation criteria in solid tumours: revised RECIST guideline (version 1.1). Eur J Cancer 45: 228-247, 2009.

12. National Cancer Institute (NCI): Common Terminology Criteria for Adverse Events (CTCAE) v4.0. https://evs.nci.nih.gov/ftp1/ CTCAE/CTCAE_4.03/. Accessed November 25, 2021.

13. Gebski V, Lagleva M, Keech A, Simes J and Langlands AO: Survival effects of postmastectomy adjuvant radiation therapy using biologically equivalent doses: A clinical perspective. J Natl Cancer Inst 98: 26-38, 2006.

14. ECOG-ACRIN cancer Research Group: ECOG Performance Status.https://ecog-acrin.org/resources/ecog-performance-status. Accessed November 25, 2021.

15. Obata S, Ogihara Y, Ohta Y, Kan T, Kanegae S, Inoue Y, Kuroiwa A, Inoue K, Yamanishi M, Hayashi T, et al: Possibility of a 'sentinel effect' in chemoradiotherapy and a new radiosensitizer injection (KORTUC). Jpn J Clin Radiol 63: 317-327, 2018 (In Japanese). 
16. U.S. Food and Drug Administration: KYMRIAH (tisagenlecleucel) https://www.fda.gov/vaccines-blood-biologics/ cellular-gene-therapy-products/kymriah-tisagenlecleucel. Accessed November 25, 2021.

17. Vaupel P: The role of hypoxia-induced factors in tumor progression. Oncologist 9 (Suppl 5): S10-S17, 2004.

18. Clevers H: The cancer stem cell: Premises, promises and challenges. Nat Med 17: 313-319, 2011.

19. Li L and Bhatia R: Stem cell quiescence. Clin Cancer Res 17: 4936-4941, 2011.

20. Takeishi S, Matsumoto A, Onoyama I, Naka K, Hirao A and Nakayama KI: Ablation of Fbxw7 eliminates leukemia-initiating cells by preventing quiescence. Cancer Cell 23: 347-361, 2013.

21. Iriuchishima $\mathrm{H}$, Takubo $\mathrm{K}$, Matsuoka $\mathrm{S}$, Onoyama I, Nakayama KI, Nojima Y and Suda T: Ex vivo maintenance of hematopoietic stem cells by quiescence induction through Fbxw7 and alpha; overexpression. Blood 117: 2373-2377, 2011.

22. Taniyama Y and Griendling KK: Reactive oxygen species in the vasculature: Molecular and cellular mechanisms. Hypertension 42: 1075-1081, 2003.

23. Pizzino G, Irrera N, Cucinotta M, Pallio G, Mannino F, Arcoraci V, Squadrito F, Altavilla D and Bitto A: Oxidative stress: Harms and benefits for human health. Oxid Med Cell Longev 2017: 8416763, 2017.

24. Kobayashi $M$ and Harada H: Hypoxic stress and HIF. Seikagaku 85: 187-195, 2013 (In Japanese).
25. Ogawa Y, Ue H, Tsuzuki K, Tadokoro M, Miyatake K, Sasaki T, Yokota N, Hamada N, Kariya S, Hitomi J, et al: New radio-sensitization treatment (KORTUC I) using hydrogen peroxide solution-soaked gauze bolus for unresectable and superficially exposed neoplasms. Oncol Rep 19: 1389-1394, 2008.

26. Miyatake K, Kubota K, Ogawa Y, Hamada N, Murata Y and Nishioka A: Non-surgical care for locally advanced breast cancer: Radiologically assessed therapeutic outcome of a new enzyme-targeting radio-sensitization treatment, Kochi Oxydol-radiation therapy for unresectable Carcinomas, Type II (KORTUC II) with systemic chemotherapy. Oncol Rep 24: 1161-1168, 2010.

27. Ogawa Y, Kubota K, Ue H, Tadokoro M, Matsui R, Yamanishi T, Hamada N, Kariya S, Nishioka A, Nakajima H, et al: Safety and effectiveness of a new enzyme-targeting radio-sensitization treatment (KORTUC II) for intratumoral injection for low-LET radio-resistant tumors. Int J Oncol 39: 553-560, 2011.

28. Chapman JD, Dugle DL, Reuvers AP, Meeker BE and Borsa J: Studies on the radio-sensitizing effect of oxygen in Chinese hamster cells. Int J Radiat Biol Relat Stud Phys Chem Med 26: 383-389, 1974.

This work is licensed under a Creative Commons

Attribution-NonCommercial-NoDerivatives 4.0 International (CC BY-NC-ND 4.0) License. 